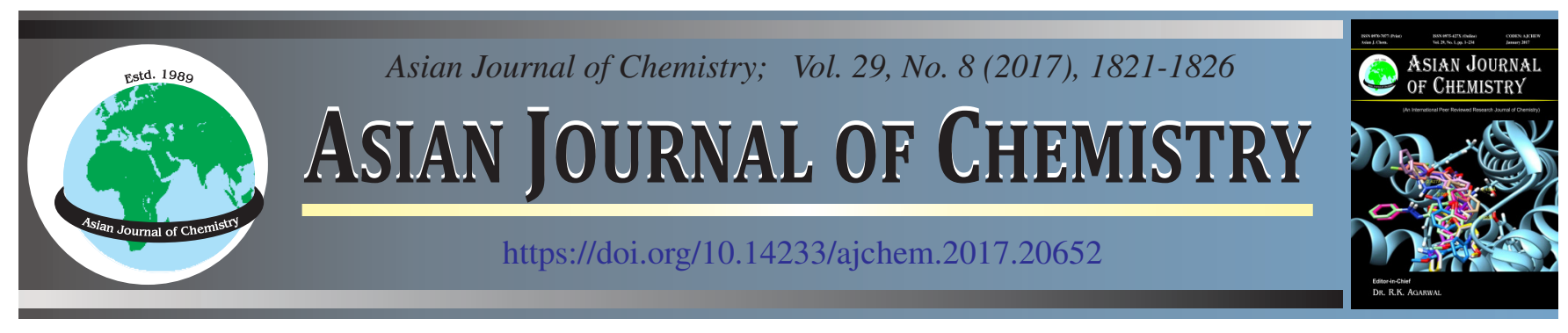

\title{
A Highly Sensitive and Rugged LC-MS/MS Method Development and Validation for Determination of Elvitegravir in Rat Plasma: Application to Pharmacokinetic Study
}

\author{
Varaprasad Adepu $^{1,2, *}$, Eswara Venkata Nagoji Kambhayatughar ${ }^{3}$ and Girija Sastry Vedula ${ }^{4}$
}

${ }^{1}$ School of Pharmaceutical Sciences and Technologies, Jawaharlal Nehru Technological University Kakinada, Kakinada-533 003, India ${ }^{2}$ Department of Pharmaceutical Analysis, Jyothishmathi Institute of Pharmaceutical Sciences, Karimnagar-505 481, India

${ }^{3}$ Department of Pharmaceutical Analysis, Srivenkateswara College of Pharmacy, Srikakulam-532 410, India

${ }^{4}$ Department of Pharmaceutical Chemistry, Andhra University, Visakhapatnam-530 003, India

*Corresponding author: E-mail: varaprasadadepu@gmail.com

\begin{abstract}
A sensitive, rugged and validated liquid chromatography-tandem mass spectrometry (LC-MS/MS) method is required for the reliable quantification of elvitegravir in rat pharmacokinetic samples. The objective of the current analytical work was to develop and validate LC-MS/MS method for the pharmacokinetic study after oral administration of elvitegravir in rats. The chromatographic separation was achieved isocratically using $5 \mathrm{mM}$ ammonium formate $(\mathrm{pH} 3.0 \pm 0.2)$ : acetonitrile (30:70 v/v) on Phenomenex Luna $5 \mu, \mathrm{C}_{18}$ column $(100$ $\mathrm{mm} \times 4.60 \mathrm{~mm}$ ) with a flow rate and total run time of $0.6 \mathrm{~mL} / \mathrm{min}$ and $4 \mathrm{~min}$, respectively. The internal standard was elvitegravir D5. The linearity plots were analyzed by using $1 / \mathrm{x}^{2}$ associated with a ten-point calibration standards. The range of concentration of elvtegravir for the method validation was 20.170 to $3428.942 \mathrm{ng} / \mathrm{mL}\left(\mathrm{r}^{2} \geq 0.9976\right)$. The retention times of elvitegravir and elvitegravir D5 were $1.05 \pm$ $0.015 \mathrm{~min}$ and $1.06 \pm 0.015 \mathrm{~min}$, respectively. The \% recovery for analyte and internal standard was $80.84 \pm 4.99$ and $85.09 \pm 3.45$, respectively. The \% mean stability for analyte was in the range of 97.84 to 105.31 . The developed and systematically validated method was effectively implemented to the pharmacokinetic analysis of rat plasma samples following oral administration.
\end{abstract}

Keywords: Elvitegravir, LC-MS/MS, Rat plasma, Pharmacokinetics, Validation.

\section{INTRODUCTION}

Elvitegravir (EVG), chemically is 6-[(3-chloro-2-fluorophenyl)methyl]-1-[(2S)-1-hydroxy-3-methylbutan-2-yl]-7methoxy-4-oxoquinoline-3-carboxylic acid (Fig. 1). Elvitegravir is a HIV-1 integrase strand transfer inhibitor (INSTI) belonging to a class of antiretroviral compounds developed by Gilead Sciences and licensed from Japan Tobacco Inc. [1,2]. Integrase is a HIV-1 encoded enzyme that is required for viral replication. Inhibition of integrase prevents the integration of HIV-1 DNA into the host genetic material, blocking the formation of the HIV-1 provirus and a broad literature review revealed that numerous analytical methods, including high performance liquid chromatography propagation of the viral infection $[3,4]$. Elvitegravir does not inhibit human topoisomerases I or II. The empirical formula of elvitegravir is $\mathrm{C}_{23} \mathrm{H}_{23} \mathrm{NO}_{5} \mathrm{FCl}$ and the molecular weight is $447.883 \mathrm{~g} / \mathrm{mol}$. Elvitegravir is a white to pale yellow solid with solubility in dimethyl sulfoxide, acetonitrile and methanol. The partition coefficient (log p) for elvitegravir is 5.5 and the $\mathrm{pKa}$ is 6.6. Elvitegravir is metabolized by enzymes as CYP 3A4, a cytochrome P450 isomer<smiles>COc1cc2c(cc1Cc1cccc(Cl)c1F)c(=O)c(C(=O)O)cn2[C@@H](CO)C(C)C</smiles>

Fig. 1. Chemical structure of elvitegravir

and glucuronosyl transferases. The trade name of elvitegravir is Stribild (fixed dose combination) or Vitekta (single pill formulation).

To evaluate further pharmacokinetic and pharmacodynamic studies of elvitegravir, a sensitive and simple analytical method is essential for the quantification of elvitegravir concentrations in plasma. For the simultaneous quantification of elvitegravir with other antiviral agents in bulk drugs and tablet 
formulations [5-11]. Aditya et al. [12] developed a reverse phase high performance liquid chromatography (RP-HPLC) method for the quantification of elvitegravir in rat plasma. This method was reported that the dispersive liquid-liquid microextraction technique was three fold more efficient on comparison with protein precipitation method. LC-MS/MS [13] have been developed and used for the simultaneous quantification of elvitegravir and ritonavir in rat plasma. UPLC-MS/MS simultaneous estimation method has been developed for quantification of elvitegravir with 15 other antiretroviral drugs in human plasma [14]. To illustrate plasma levels of elvitegravir with rilpivirine (RPV) in HIV patients, Aouri et al. [15] reported a LC-MS/MS method with the stable isotopic labeled compounds RPV-13C6 and elvitegravir D5 as corresponding internal standards. It is vastly suggested to use a stable isotope-labeled analog for quantitative LC-MS/MS analysis. However, the reported methods have higher limits of detection and also run times. Hence a more sensitive and highly specific LC-MS/MS rugged method was developed and validated to quantify the elvitegravir in rat plasma and its application to pharmacokinetics study.

\section{EXPERIMENTAL}

Elvitegravir (analyte), elvitegravir D5 (internal standard) were obtained as gift samples from Gilead Sciences Inc USA, ammonium formate and acetonitrile are of HPLC grade and purchased from Merck Pvt. Ltd Mumbai, India, tert-butyl methyl ether $(t-\mathrm{BME})$ is procured from Thomas Baker Chemicals Pvt. Ltd., Mumbai, Inida.

Chromatographic and mass spectrometry conditions: The chromatographic separation was achieved isocratically using a high performance liquid chromatography separation module coupled with Quattro Premier XE ${ }^{\circledR}$ mass spectrometer comprising two LC-20AD pumps, a cooling autosampler (SIL $20 \mathrm{AC}$ ), a column oven temperature control (CTO-20AC) and a CBM 20 A controller. With the mobile phase of $5 \mathrm{mM}$ ammonium formate $(\mathrm{pH} 3.0 \pm 0.2)$ :acetonitrile $(30: 70 \mathrm{v} / \mathrm{v})$ at a flow rate of $0.6 \mathrm{~mL} / \mathrm{min}$ on Phenomenex Luna $5 \mu, \mathrm{C} 18$ column $(100 \mathrm{~mm} \times 4.60 \mathrm{~mm})$ with a total run time of $4.0 \mathrm{~min}$. Elvitegravir D5 was used as an internal standard. The adjusted column temperature was $20{ }^{\circ} \mathrm{C}$.

The injection volume was $20 \mu \mathrm{L}$. The method was validated over a concentration range of 20.170 to $3428.942 \mathrm{ng} / \mathrm{mL}$ with the correlation coefficient $\geq 0.9976$ by using $1 / \mathrm{x}^{2}\left(1 /\right.$ conc $\left.^{2}\right)$ weighted least square regression analysis of standard plots associated with ten-point calibration standards. The retention times of elvitegravir and elvitegravir D5 were $1.05 \pm 0.015$ $\mathrm{min}$ and $1.06 \pm 0.015 \mathrm{~min}$, respectively. Electrospray ionization was performed using Nebulizer gas 50 \& heater gas 40, curtain gas flow rate 25 and collision activated dissociation 6; ion spray voltage (IS) 5500 ; temperature $500{ }^{\circ} \mathrm{C}$; pause time 5 $\mathrm{ms}$; collision gas - nitrogen; for elvitegravir and elvitegravir D5:- dwell time $100 \mathrm{~ms}$; declustering potential $50 \mathrm{~V}$; collision energy 28; collision cell exit potential 10V; entrance potential $10 \mathrm{~V}$. The MRM transitions for analyte and internal standard were $449.9 \rightarrow 348$ and $454.9 / 353$ respectively.

Preparation of standards and quality control samples: Individual stock $(1 \mathrm{mg} / \mathrm{mL})$ solutions of elvitegravir and elvite- gravir D5 were prepared by dissolving pure compound of elvitegravir and elvitegravir D5 in sufficient HPLC grade methanol and were stored at $2-8{ }^{\circ} \mathrm{C}$ in the refrigerator until further dilution. All the required intermediate and working standards of elvitegravir and elvitegravir D5 were prepared from the respective stock solutions by diluting with dilution fluid comprising mixture of water and methanol at the ratio of 30:70 v/v. Calibration standards were prepared between 20.170 to $3428.942 \mathrm{ng} / \mathrm{mL}$ of using ten concentration levels. Quality control standards of three different levels low (LQC) (30.00 $\mathrm{ng} / \mathrm{mL})$, medium (MQC) $(2400.25 \mathrm{ng} / \mathrm{mL})$ and high (HQC) ( $3900.40 \mathrm{ng} / \mathrm{mL}$ ) were also prepared. All these stock solutions, calibration standards and quality control samples were stored at $4 \pm 2{ }^{\circ} \mathrm{C}$.

Sample preparation: To an aliquot of $200 \mu \mathrm{L}$ of plasma sample, $25 \mu \mathrm{L}$ of internal standard was added. To the resulting mixture, $200 \mu \mathrm{L}$ of extraction buffer ( $5 \mathrm{mM}$ ammonium formate) was added, mixed well and vortexed for few seconds, then $1 \mathrm{~mL}$ of extraction solvent tert-butyl methyl ether was added, vortexed again for $5 \mathrm{~min}$, centrifuged and supernatant was collected for evaporation and reconstituted with $200 \mu \mathrm{L}$ of mobile phase.

Method validation: The developed method was systematically validated according to FDA guidelines [16]. The specificity of the method was assessed by testing the blank plasma from at least six different rats. Blank and lower limit of quantification (LLOQ) were processed from each of the six different rats as per the method described under sample preparation section. To determine the specificity, the percentage CV and percentage accuracy were considered after injecting six processed LLOQ samples

The calibration curve for the method was constructed by plotting the peak area ratio of elvitegravir: elvitegravir D5 (IS) against the nominal concentration of calibration standards in rat plasma. The linearity of the method was determined by using a $1 / x^{2}$ weighted least square regression analysis of standard plots associated with a ten-point standard curve.

The intra and inter-day precision and accuracy were carried out by injecting six samples at each quality control level (LLOQ, LQC, MQC1, MQC2 and HQC) and analyzing against the standard calibration curve. The \% CV and \% accuracy at each quality control level were calculated.

Unextracted aqueous solutions of LQC, MQC1, MQC2 and HQC were prepared. Recovery of elvitegravir and elvitegravir D5 from rat plasma sample was determined by comparing the mean peak area response of elvitegravir in the extracted sample to the mean peak area response of the elvitegravir in the corresponding aqueous unextracted sample solutions.

The ruggedness of an analytical method is a measure of its capacity to remain unaffected by small, but predetermined variations in operating conditions. The ruggedness of the developed method was assessed by analyzing the quality control samples by the different analysts and different equipment from the same make and model with some purposeful changes in the conditions. The ruggedness test should meet the acceptance criteria for linearity and intra-batch accuracy and precision.

The matrix effect of the method was inspected by injecting six LLOQ samples prepared from different rat plasma. These 
responses were analyzed using freshly prepared standard calibration curve and at least two sets of LLOQ samples qualifying were prepared in previously screened rat plasma. The \% $\mathrm{CV}$ of LLOQ and $\mathrm{S} / \mathrm{N}$ ratio of mean LLOQ for different batches were calculated.

Re-injection reproducibility determined by re-injecting the LQC, MQC1, MQC2 and HQC of the precision and accuracy batch following a break of $18 \mathrm{~h} 40 \mathrm{~min}$ and examined against formerly analyzed calibration curve. The $\% \mathrm{CV}$ of the formerly found concentrations of quality control samples and those acquired after re-injection were estimated.

Dilution integrity makes sure that a known concentration above the ULOQ can be effectively diluted and analyzed to make an actual concentration outcome with adequate accuracy. Six replicate of 1:2 and 1:5 diluted ULOQ samples were arranged and concentrations were calculated using suitable dilution factor.

The stability of elvitegravir and the internal standard in plasma was evaluated in order to determine the stock solution solubility, stability of the analyte in matrix, freeze and thaw stability, bench top stability and in-injector stability in auto sampler, dry extract stability. Stability studies were conducted by examining the rat plasma samples at two quality control levels namely LQC and HQC, each at six replicates after storage at different time and temperature conditions. Samples were spiked at each quality control levels to study the long term stability of elvitegravir in rat plasma.

Application to pharmacokinetic study in rats: The developed and systematically validated analytical method was effectively implemented to measure elvitegravir levels in rat pharmacokinetic samples. The study was carried out as per current GCP guidelines [16-18] and was also approved by Institutional Ethical Committee, Care College of Pharmacy, Warangal-506006, Telangana State, India. Total 12 male Wistar rats (170-210 g) were selected and divided into two groups each group with equal animals. The dose of $15 \mathrm{mg} / \mathrm{kg}$ body weight of elvitegravir was given orally with the aid of oral feeding needle. The dose was arranged by adding suitable quantity of elvitegravir in sterile normal saline containing $0.5 \%$ sodium carboxy methyl cellulose. The blood samples of $0.5 \mathrm{~mL}$ were collected via retro arbitral vein at predetermined time intervals $(0,0.5,1,2,4,6,8,10,12,14,16,18,24$ h) for analysis. The blood samples were collected into separate tubes containing an anticoagulant $\mathrm{K}_{2}$ EDTA. Samples were centrifuged at $3000 \mathrm{rpm}$ for the separation of plasma within the temperature range of $2-8{ }^{\circ} \mathrm{C}$. The processed pharmacokinetic study plasma samples were stored at $-30{ }^{\circ} \mathrm{C}$ until analysis is completed. After analysis the pharmacokinetic factors were calculated with help of KINETICA software version 5.0 trial version Adept Scientific limited.

\section{RESULTS AND DISCUSSION}

While the course of validation, blank rat plasma samples from 14 different lots [8 normal, $2(0.5 \%)$ haemolyzed, 2 (1\%) haemolyzed and 2 lipemic] were evaluated. During the specificity run, six LLOQ standards were extracted and injected. The responses for the blank plasma from the 14 different lots were compared to the LLOQ standard mean area of elvitegravir and internal standard. No significant peak was shown at the retention time of the elvitegravir and the elvite-gravir D5 in blank rat plasma as compared to the LLOQ standard. No significant injector carry over is observed for elvitegravir and internal standards. Fig. 2 shows the typical chromatograms of blank, blank with ISTD and LLOQ.
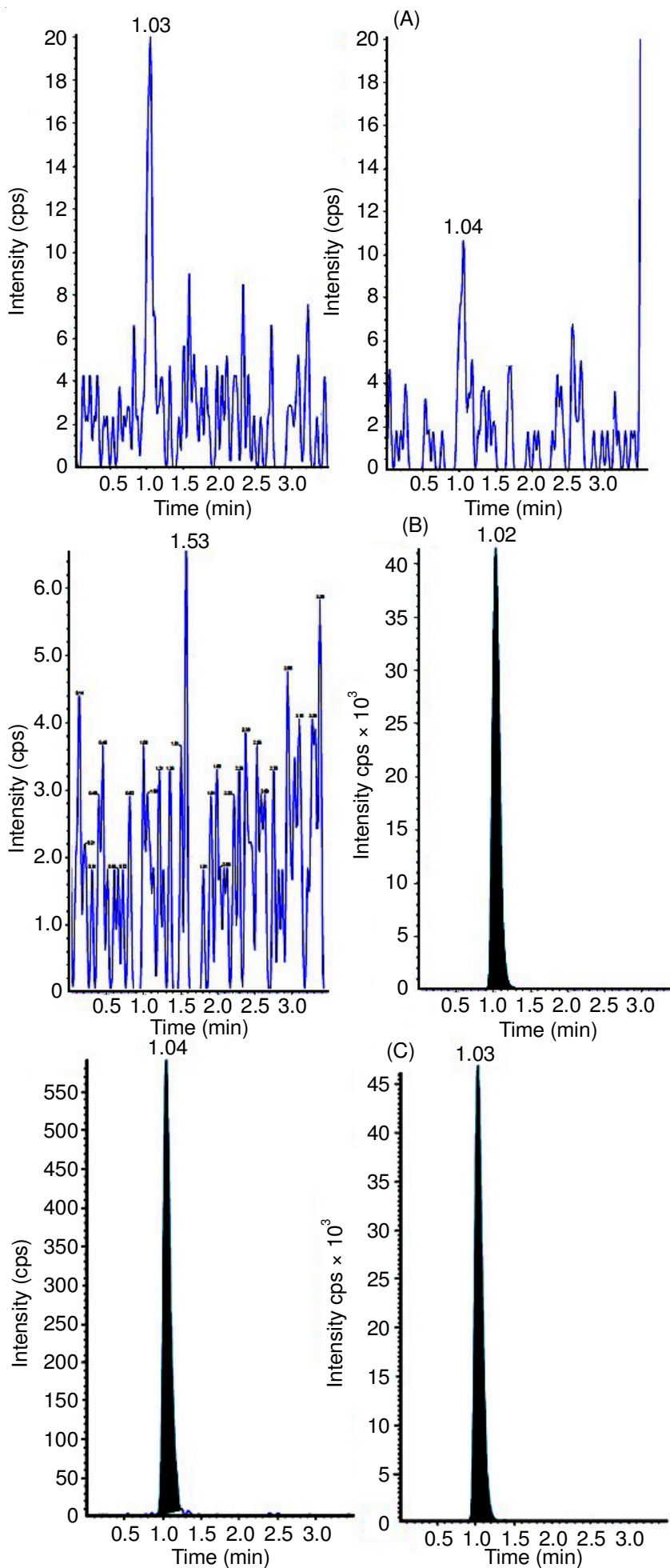

Fig. 2. Representative chromatograms of (A) blank, (B) blank with ISTD (C) LLOQ

Calibration curves were accurate and precise for elvitegravir over 20.170 to $3428.942 \mathrm{ng} / \mathrm{mL}$ calibration range. All 
the calibration curves analyzed throughout the line of validation were established to be linear $\left(r^{2} \geq 0.9976\right)$. Back calculations were made for the determination of elvitegravir from each calibration curve. The mean $\%$ nominal concentrations were found to be between 94.66 to $111.97 \%$, which were within the limit $(85-115 \%$ for all calibration standards except lowest calibration standard and 80-120\% for lowest calibration standard). The calculated $\% \mathrm{CV}$ for all calibration standards were established to be in the range of 1.36 to $7.01 \%$. The obtained values were within accepted range (for all calibration standards, it should be $\leq 15 \%$, except for lowest calibration standard and for the lowest calibration standard should be $\leq 20 \%$ ).

Intra batch/run/day accuracy and precision: Intra batch accuracy and precision assessment were evaluated by examining 3 sets of calibration curves and five levels of quality control samples; each set of calibration curve consists of 6 replicates of the each quality control level namely, LLOQQC, LQC, M1QC, M2QC and HQC. The results were represented in Table-1. The \% CV intra batch accuracy and precision was less than

\begin{tabular}{|c|c|c|c|}
\hline \multicolumn{4}{|c|}{$\begin{array}{c}\text { TABLE-1 } \\
\text { INTER (BETWEEN-BATCH/RUN/DAY) \& INTRA } \\
\text { BATCH (WITHIN BATCH/RUN/DAY) ACCURACY } \\
\text { AND PRECISION FOR DETERMINATION OF } \\
\text { ELVITEGRAVIR LEVELS IN RAT PLASMA }\end{array}$} \\
\hline Sample & $\begin{array}{l}\text { Mean conc. found } \\
(\mathrm{ng} / \mathrm{mL}) \pm \mathrm{SD}\end{array}$ & $\begin{array}{c}\text { Mean } \\
\text { accuracy }(\%) \\
\end{array}$ & $\mathrm{CV}(\%)$ \\
\hline \multicolumn{4}{|c|}{ Intra-day $(\mathrm{n}=6)$} \\
\hline \multicolumn{4}{|c|}{ P\&A batch I } \\
\hline LLOQ & $19.72 \pm 0.79$ & 98.33 & 3.99 \\
\hline LQC & $61.56 \pm 0.55$ & 102.31 & 0.89 \\
\hline MQC 2 & $816.85 \pm 6.12$ & 101.81 & 0.75 \\
\hline MQC 1 & $1657.26 \pm 24.64$ & 97.20 & 1.49 \\
\hline HQC & $2625.62 \pm 48.65$ & 100.69 & 1.85 \\
\hline \multicolumn{4}{|c|}{ P\&A batch II } \\
\hline LLOQ & $18.95 \pm 1.14$ & 94.47 & 6.05 \\
\hline LQC & $60.10 \pm 0.90$ & 101.36 & 1.47 \\
\hline MQC 2 & $811.79 \pm 8.38$ & 101.18 & 1.03 \\
\hline MQC 1 & $1673.34 \pm 14.32$ & 98.14 & 0.86 \\
\hline HQC & $2676.51 \pm 30.72$ & 102.64 & 1.15 \\
\hline \multicolumn{4}{|c|}{ P\&A batch III } \\
\hline LLOQ & $21.05 \pm 2.79$ & 104.93 & 13.24 \\
\hline LQC & $58.86 \pm 1.10$ & 97.82 & 3.39 \\
\hline MQC 2 & $809.89 \pm 33.20$ & 100.94 & 4.10 \\
\hline MQC 1 & $1591.29 \pm 10.82$ & 93.33 & 0.68 \\
\hline HQC & $2689.30 \pm 110.53$ & 103.13 & 4.11 \\
\hline \multicolumn{4}{|c|}{ Inter day $(\mathrm{n}=24)$} \\
\hline LLOQ & $21.05 \pm 2.79$ & 104.93 & 13.24 \\
\hline LQC & $58.86 \pm 1.99$ & 97.82 & 3.39 \\
\hline MQC 2 & $809.88 \pm 33.20$ & 100.94 & 4.10 \\
\hline MQC 1 & $1591.29 \pm 10.82$ & 93.33 & 0.68 \\
\hline HQC & $2689.30 \pm 110.53$ & 103.13 & 4.11 \\
\hline
\end{tabular}

$15 \%$ for all quality control samples except LLOQQC and it was less than $20 \%$ for LLOQQC.

Inter batch/run/day accuracy and precision: Inter batch accuracy and precision evaluation were assessed by analyzing 4 sets of calibration curves and levels of quality control samples. Each set of calibration curves consists of 6 replicates of each quality control level namely, LLOQQC, LQC, M1QC, M2QC and HQC. The results were represented in Table-1. The \% CV intra-batch/interbatch accuracy and precision was less than $15 \%$ for all quality control samples except LLOQQC and it was less than $20 \%$ for LLOQQC.

Percentage recovery: The percentage recovery of elvitegravir was determined by comparing the mean peak area of elvitegravir in extracted LQC, MQC1, MQC2 and HQC samples with freshly prepared unextracted LQC, MQC1, MQC2 and HQC samples respectively. The \% recovery for analyte and internal standard was $80.84 \pm 4.99$ and $85.09 \pm 3.45$, respectively. The $\% \mathrm{CV}$ for mean recovery of analyte was less than $15 \%$. The $\% \mathrm{CV}$ for the extracted and unextracted internal standard was less than $25 \%$.

Ruggedness: The ruggedness experiment was conducted by injecting samples of previously passed precision accuracy batch on a different column with the same make and specification. Concentrations were determined to calculate precision and accuracy. Table- 2 shows that elvitegravir results were found to be reproducible on a different column with the same make and specification. The \% CV for all quality control samples was less than $15 \%$, except LLOQQC and it was less than $20 \%$ for LLOQQC. The \% mean concentration of all quality control samples was within the range $(85-115 \%)$ except LLOQQC and it was 80-120\% for LLOQQC.

Dilution integrity: The dilution integrity experiment was carried out at six replicate of two times diluted (1 in 2 dilution) and five times diluted ( 1 in 5 dilution) of approx. 1.5X ULOQ samples were arranged and concentrations were calculated against calibration curve and compared with nominal concentrations. The $\%$ nominal quantities were within the limit (85$115 \%)$

Re-injection reproducibility: The whole batch re-injection reproducibility experiment was evaluated using samples of previously passed precision and accuracy batch were placed in the auto sampler and re-injected again approximately $18 \mathrm{~h}$ $40 \mathrm{~min}$ after initial analysis. Concentrations were determined to calculate precision and accuracy after re-injection. The $\%$ $\mathrm{CV}$ for all re-injected samples was within the limit (less than $15 \%$ ), except LLOQQC and it was less than $20 \%$ for LLOQQC. The $\%$ mean nominal concentration for all re-injection samples were within the range (85-115\%).

TABLE-2

RUGGEDNESS USING A DIFFERENT COLUMN IN RAT PLASMA

\begin{tabular}{ccc|ccc|cc|ccc|cc}
\hline & \multicolumn{2}{c|}{ LLOQQC } & \multicolumn{2}{c|}{ LQC } & \multicolumn{2}{c|}{ MQC1 } & \multicolumn{2}{c|}{ MQC2 } & \multicolumn{2}{c}{ HQC } \\
\cline { 2 - 12 } & C-01 & C-02 & C-01 & C-02 & C-01 & C-02 & C-01 & C-02 & C-01 & C-02 \\
\hline N & 6 & 6 & 6 & 6 & 6 & 6 & 6 & 6 & 6 & 6 \\
Mean & 19.07 & 20.50 & 61.00 & 64.72 & 811.79 & 818.47 & 1673.34 & 1680.78 & 2676.51 & 2734.56 \\
SD & 1.24 & 0.9812 & 0.90 & 1.167 & 8.38 & 11.17 & 14.33 & 16.76 & 30.72 & 27.40 \\
CV (\%) & 6.49 & 4.79 & 1.47 & 1.80 & 1.03 & 1.37 & 0.86 & 1.00 & 1.15 & 1.00 \\
NOM (\%) & 95.07 & 102.20 & 101.36 & 107.55 & 101.18 & 102.01 & 98.14 & 98.58 & 102.64 & 104.87 \\
\hline
\end{tabular}


Stability analysis: Stock solution stability study results were analyzed by comparing the peak areas of newly arranged stock solutions (comparison samples) with stability stock solutions.

Short term stock solution stability at room temperature in diluent (s): The stock solutions of elvitegravir and elvitegravir D5 were newly arranged and samples of stocks were placed at room temperature for approximately $12 \mathrm{~h} 25 \mathrm{~min}$. Aqueous equivalent to LQC \& HQC concentrations of elvitegravir along with internal standards (elvitegravir D5) were prepared from the stability samples and comparison samples (stocks stored at $0-10{ }^{\circ} \mathrm{C}$ ). Six replicates injected from each vial of both stability and freshly prepared samples and their respective peak areas were compared to analyze $\%$ mean change for the period of stability study. Elvitegravir and elvitegravir D5 stock solutions were established to be stable at room temperature for approximately 12 h $25 \mathrm{~min}$ in diluent 1 and diluent 2 . Results are presented in Table-3. Long term stock solution stability at 0$10{ }^{\circ} \mathrm{C}$ : The stock solutions of the elvitegravir and elvitegravir D5 were arranged and placed in the refrigerator at $0-10{ }^{\circ} \mathrm{C}$ for 27 days and 31 days in diluents-1, respectively. The elvitegravir and elvitegravir D5 were prepared and stored in the refrigerator at $0-10{ }^{\circ} \mathrm{C}$ for 27 days and 26 days in diluent-2. The above stored stocks and working solutions of elvitegravir and elvitegravir D5 were compared against fresh stock solutions, 6 replicates injected from each vial of both stability and comparison samples (freshly prepared samples) and area response was compared for elvitegravir and elvitegravir D5 to determine mean $\%$ change during the stability period. The results indicate that the stock solutions of elvitegravir and elvitegravir 5 were found to be stable for 31 days.

The working solution of elvitegravir was established to be stable for 27 days in diluent- 2 and working solution of elvitegravir D5 was found to be stable for 26 days in diluent2 in the refrigerator at $0-10{ }^{\circ} \mathrm{C}$. Results are presented in Table- 3 .

Bench top stability at room temperature: Six samples of each LQC and HQC spiked, were retrieved from deep freezer and were placed at room temperature for approximately $6 \mathrm{~h}$ $30 \mathrm{~min}$ and were processed and investigated by the side of newly arranged calibration standards and LQC and HQC samples. Concentrations were determined to calculate mean \% change for the period of stability study. Elvitegravir were established to be stable in LQC and HQC samples for approximately $6 \mathrm{~h} 30 \mathrm{~min}$.

Dry extract stability: The stability of elvitegravir (after extraction and sample preparation in the dry extract condition) during storage in the refrigerator $\left(0-10{ }^{\circ} \mathrm{C}\right)$ was documented by comparison of the concentrations of extracted replicates to the comparison samples (freshly prepared LQC and HQC) concentrations. The six sets of extracted plasma quality control samples (LQC and high HQC) for elvitegravir were kept in a refrigerator at $0-10{ }^{\circ} \mathrm{C}$ for approximately $20 \mathrm{~h} 15 \mathrm{~min}$. They were then analyzed using a freshly prepared calibration standards and freshly prepared comparison samples.

Freeze and thaw stability at $-70 \pm 15^{\circ} \mathrm{C}$ : LQC and HQC samples were prepared, frozen at $-70 \pm 15^{\circ} \mathrm{C}$. Six samples from each quality control level (stability samples) were subjected to four freeze and thaw cycles. These stability samples were practiced and examined by the side of newly arranged calibration standards and LQC and HQC samples (comparison samples). Concentrations were determined to calculate mean $\%$ change after four cycles. Elvitegravir was established to be stable in selected quality control samples after four cycles.

In-injector stability in auto sampler at $10{ }^{\circ} \mathrm{C}$ : Three sets of LQC and HQC samples were processed (each set contain 6 samples at each level). The 1 st set of processed samples were analyzed as $0.00 \mathrm{~h}$. The $2 \mathrm{nd}$ and $3 \mathrm{rd}$ sets were kept in auto sampler for approx. $60 \mathrm{~h} 20 \mathrm{~min}$ at $10^{\circ} \mathrm{C}$. 2nd set was analyzed by the side of newly arranged calibration standard samples and compared against set 1-0.00 h (comparison samples). Concentrations were calculated to determine mean $\%$ change during the stability period.

Long term stability of analyte in rat plasma: Samples were spiked at LQC and HQC levels, aliquoted and frozen at $-70 \pm 15^{\circ} \mathrm{C}$. Six samples of each concentration were taken out. Stability samples were processed and analyzed along with freshly prepared calibration standards, LQC and HQC samples (comparison samples). Concentrations were calculated for elvitegravir to determine mean $\%$ change during the stability period and found to be stable at $-70 \pm 15^{\circ} \mathrm{C}$ for 14 days. The stability temperature and time conditions for all types of stability studies were represented in Table-3. In short term stock solution stability at room temperature and long term stock solution stability at $0-10{ }^{\circ} \mathrm{C}$, the $\%$ change and the mean $\%$ stability of tested quality control samples when compared against control samples was not more than $5 \%$ and within the 95-105\%, respectively for elvitegravir and elvitegravir D5. In stability study of elvitegravir in biological matrix at room temperature and stability study as dry extract at $0-10{ }^{\circ} \mathrm{C}$, freeze-thaw stability study at $-70 \pm 15^{\circ} \mathrm{C}$, auto sampler stability at $10{ }^{\circ} \mathrm{C}$ and long term stability in biological matrix at $-70 \pm 15^{\circ} \mathrm{C}$, the $\%$ change and the mean $\%$ stability of tested quality control samples when

TABLE-3

STABILITY STUDY CONDITIONS AND \% MEAN STABILITY RESULTS

\begin{tabular}{|c|c|c|c|c|}
\hline \multirow{3}{*}{ Stability study } & \multirow{3}{*}{ Condition } & \multirow{3}{*}{$\mathrm{n}$} & \multicolumn{2}{|c|}{ Mean stability (\%) } \\
\hline & & & \multicolumn{2}{|c|}{ Elvitegravir } \\
\hline & & & HQC & LQC \\
\hline Short term stock solution stability & Storage at room temperature for $15 \mathrm{~h} 45 \mathrm{~min}$ & & 100.63 & 100.60 \\
\hline Long term stock solution stability & Storage at $0-10^{\circ} \mathrm{C}$ temperature for 31 days & & 100.47 & 102.56 \\
\hline Long term stock solution stability & Storage at $-70 \pm 5^{\circ} \mathrm{C}$ temperature for 14 days & & 103.75 & 97.21 \\
\hline Stability of analyte in blood & Storage at room temperature for $6 \mathrm{~h} 30 \mathrm{~min}$ & 6 & 98.48 & 98.68 \\
\hline Dry extract stability & Storage at $0-10{ }^{\circ} \mathrm{C}$ for a period of $26 \mathrm{~h} 34 \mathrm{~min}$ & & 99.81 & 97.84 \\
\hline Auto sampler stability & Storage at $10^{\circ} \mathrm{C}$ for $60 \mathrm{~h} 20 \mathrm{~min}$ & & 99.23 & 102.05 \\
\hline
\end{tabular}


compared against control samples was not more than $15 \%$ and within the $85-115 \%$, respectively for elvitegravir (Table-3)

Application of pharmacokinetic study in rat plasma: The developed LC-MS/MS method was successfully applied for determination of the elvitegravir concentration after oral administration of $15 \mathrm{mg} / \mathrm{kg}$ body weight in $6 \mathrm{Wistar}$ rats. The standard calibration curve was used for estimation of the elvitegravir in the pharmacokinetic study plasma samples. Rat blood samples of approximately $0.5 \mathrm{~mL}$ were collected at different predetermined time periods $(0,0.5,1,2,4,6,8,10,12$, 18 and $24 \mathrm{~h}$ ) after oral administration of elvitegravir. The plasma sample was extracted as described in the previous section. Fig. 3 shows the plasma concentration-time profile of elvitegravir in Wistar rat plasma. The corresponding calculated all possible pharmacokinetic parameters were represented in Table-4.

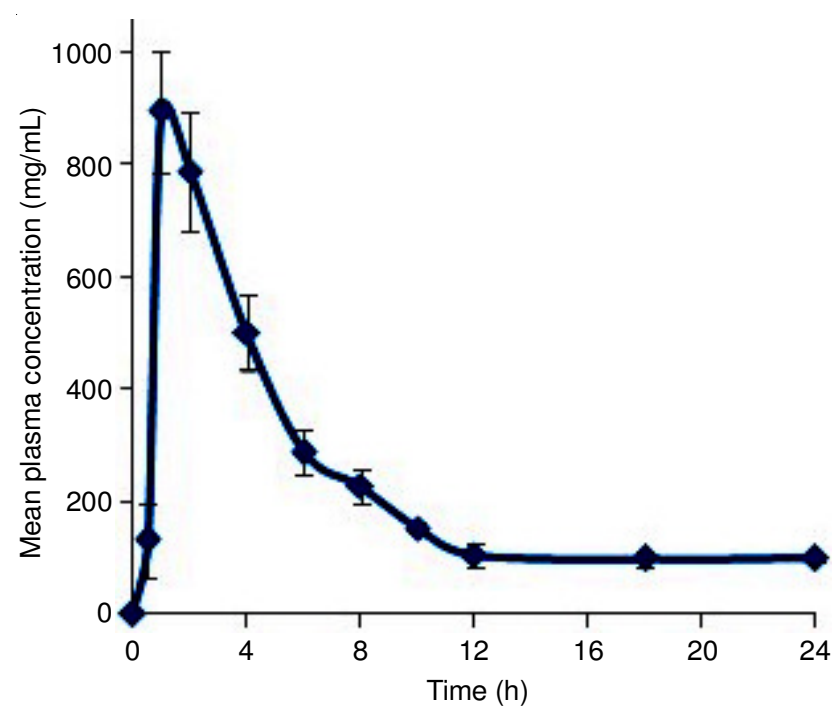

Fig. 3. Mean plasma concentration-time curve of elvitegravir in wistar rats after oral administration of pure elvitegravir at a dose of $15 \mathrm{mg} / \mathrm{kg}$ body weight $(\mathrm{n}=6)$

\begin{tabular}{|c|c|}
\hline \multicolumn{2}{|c|}{$\begin{array}{c}\text { TABLE-4 } \\
\text { SUMMARY OF PHARMACOKINETICS OF ELVITEGRAVIR } \\
\text { PURE FROM SIX HEALTHY MALE WISTAR RATS } \\
\text { AFTER ORAL DOSE OF } 15 \mathrm{mg} / \mathrm{kg} \text { BODY WEIGHT }\end{array}$} \\
\hline PK parameter & Pure elvitegravir \\
\hline $\mathrm{C}_{\max }(\mathrm{ng} / \mathrm{mL})$ & $894.788 \pm 108.075$ \\
\hline $\mathrm{T}_{\max }(\mathrm{h})$ & $1.00 \pm 0.00$ \\
\hline $\mathrm{AUC}_{0-24}\left(\mathrm{ng} \mathrm{mL}^{-1} \mathrm{~h}\right)$ & $4300.474 \pm 461.356$ \\
\hline $\mathrm{AUC}_{0-\infty}\left(\mathrm{ng} \mathrm{mL}^{-1} \mathrm{~h}\right)$ & $4836.581 \pm 551.713$ \\
\hline $\mathrm{t}_{1 / 2}(\mathrm{~h})$ & $3.382 \pm 0.159$ \\
\hline $\operatorname{MRT}(\mathrm{h})$ & $5.722 \pm 0.304$ \\
\hline
\end{tabular}

\section{Conclusion}

A simple method using LC-MS/MS for the determination of elvitegravir in rat plasma employing simple liquid-liquid extraction was developed. The method was rapid, precise, specific and sensitive and also demonstrates good accuracy and precision. It is believed that this high throughput method could provide a useful tool for the determination of elvitegravir in rat plasma. The precision and mean accuracy for the analytical method are within the acceptable limits and the samples may be diluted, if required. Recoveries were consistent for all quality samples (LQC, MQC1, MQC2 and HQC). The method was proved to be rugged when used a different column, yielding reproducible results for elvitegravir. Results were accurate and repeated after re-injection for $18 \mathrm{~h} 40 \mathrm{~min}$. Stability tests proved the analyte was stable at different conditions like auto sampler (60 h $20 \mathrm{~min}$ at $10^{\circ} \mathrm{C}$ ), bench top stability (6 h $30 \mathrm{~min}$ ), dry extract stability (at $0-10{ }^{\circ} \mathrm{C}$ for $20 \mathrm{~h} 15 \mathrm{~min}$ ), freeze thaw stability (at $-70 \pm 15^{\circ} \mathrm{C}$ ). Stock solutions of elvitegravir and elvitegravir D5 were stable at room temperature for about $12 \mathrm{~h}$ $25 \mathrm{~min}$. The established method was successfully applied to a pharmacokinetics study in rat and to assess the plasma concentration of elvitegravir.

\section{ACKNOWLEDGEMENTS}

The authors are thankful to Dr. Kiran Gangarapu, Head Deparment of Pharmaceutical Analysis and Quality Assurance, School of Pharmacy, Anurag Group of Institutions, Hyderabad, India, for proof reading of the complete manuscript.

\section{REFERENCES}

1. T. Wills, V. Vega and A. Elvitegravir, Expert Opin. Investig. Drugs, 21, 395 (2012); https://doi.org/10.1517/13543784.2012.658914.

2. $\quad$ S.L. Karmon and M. Markowitz, Drugs, 73, 213 (2013); https://doi.org/10.1007/s40265-013-0015-5.

3. D. Esposito and R. Craigie, Adv. Virus Res., 52, 319 (1999); https://doi.org/10.1016/S0065-3527(08)60304-8.

4. D.J. Hazuda, P. Felock, W. Witmer, A. Wolfe, K. Stillmock, J.A. Grobler, A. Espeseth, L. Gabryelski, W. Schleif, C. Blau and M.D. Miller, Science, 287, 646 (2000); https://doi.org/10.1126/science.287.5453.646.

5. M.R. Nagasarapu and G.S. Dannana, Indian J. Pharm. Educ. Res., 50, 205 (2016); https://doi.org/10.5530/ijper.50.1.25.

6. P.P. Rao, D.M. Reddy and D. Ramachandran, World J. Pharm. Sci., 2, 1822 (2014).

7. V.V. Ravindrababu, P.K. Sharma and I. Singhvi, Asian J. Chem., 26, 6233 (2014); https://doi.org/10.14233/ajchem.2014.17196.

8. R.R. Jampala, V.K. Kumar and A. Raju Nemala, Pharm. Methods, 5, 7 (2014); https://doi.org/10.5530/phm.2014.1.2.

9. P. Swetha, V.V.S. Rajendra Prasad, M.B. Raju and N.S. Kumar, IndoAm. J. Pharm. Res., 3, 4697 (2013).

10. S.P. Parthi, K.B. Aher and G.B. Bhavar, Int. J. Pharm. Sci., 6, 369 (2015).

11. K. Sureshbabu, S.S. Vardhan, B. Koteswararao and C. Rambabu, Pharm. Lett., 7, 205 (2015).

12. A. Aditya, P.R. Dande, S. Deshpande and R. Nageswara Rao, J. Anal. Bioanal. Tech., 5, 146 (2014).

13. R.N. Rao, K.G. Prasad, K.V.S. Kumar and B. Ramesh, Anal. Methods, 5, 6693 (2013); https://doi.org/10.1039/c3ay41346b.

14. Z. Djerada, C. Feliu, C. Tournois, D. Vautier, L. Binet, A. Robinet, H. Marty, C. Gozalo, D. Lamiable and H. Millart, J. Pharm. Biomed. Anal., 86, 100 (2013); https://doi.org/10.1016/j.jpba.2013.08.002.

15. M. Aouri, A. Calmy, B.A. Hirschel, A. Telenti, T. Buclin, M. Cavassini, A. Rauch and L.A. Decosterd, J. Mass Spectrom., 48, 616 (2013); https://doi.org/10.1002/jms.3200.

16. Guidance for Industry: Bioanalytical Method Validation, U.S. Department of Health and Human Services, Food and Drug Administration, Center for Drug Evaluation and Research (CDER), Center for Biologics Evaluation and Research (CBER), May (2001).

17. Guidance for Industry Food-Effect Bioanalytical and Fed Bioequivalence Studies, U.S. Department of Health and Human Services, Food and Drug Administration, Centre for Drug Evaluation and research (CDER), December (2002).

18. Guidance for Industry Bioavailability and Fed Bio-equivalence Studies for Orally Administered Drug Products-General Consideration, U.S. Department of Health and Human Services, Food and Drug Administration, Centre for Drug Evaluation and research (CDER), March (2003). 